Scientific Visualization, 2020, volume 12, number 5, pages 165 - 173, DOI: 10.26583/sv.12.5.12

\title{
Automated construction of a stereo image for results of math- ematical modeling using the Multi-view StereoMaker software system
}

\author{
S.V. Andreev ${ }^{1}$ A.E. Bondarev², N.A. Bondareva3, K.A. Donskoy 4, V.A. Galaktionov5 \\ Keldysh Institute of Applied Mathematics RAS \\ ${ }^{1}$ ORCID: 0000-0001-8029-1124, esa@keldysh.ru \\ 2 ORCID: 0000-0003-3681-5212, bond@keldysh.ru \\ 3 ORCID: 0000-0002-7586-903X, niki.99@mail.ru \\ 4 ORCID: 0000-0001-7597-1117, konstantindonskoy1990@gmail.com \\ 5 ORCID: 0000-0001-6460-7539, vlgal@gin.keldysh.ru
}

\begin{abstract}
$\underline{\text { Abstract }}$
This work presents the Multi-view StereoMaker software package, which allows making automatic construction of a stereo image for several objects and accompanying information to them using multi-view technology. Multi-view StereoMaker software complex works with arbitrary $3 \mathrm{D}$ objects, representing both the results of calculations of mathematical modeling problems, and user-defined images.

The construction of the display of scientific research results in stereo mode is implemented for an autostereoscopic monitor. An autostereoscopic monitor allows viewing stereo images without glasses while providing quality that rivals that of a classic $3 \mathrm{D}$ projection stereo system. This work continues the cycle of research carried out at the Keldysh Institute of Applied Mathematics. The research is aimed at developing technologies of constructing stereo images and animations for presenting the results of scientific computations on classical stereo devices and autostereoscopic monitors.

A description of the main functions and capabilities of the first version of the developed system is given. The results of the operation of the Multi-view StereoMaker software complex are presented.
\end{abstract}

Keywords: stereo images, autostereoscopic monitor, multi-view, software.

\section{Introduction}

The emergence, development and gradual spread of stereo equipment provides an opportunity for research in the field of stereo-animation technologies and their application in scientific developments. The construction of stereo-animation representations of scientific research results using such stereo devices opens up completely new possibilities the significance and relevance of which can hardly be underestimated.

When working with a flat image of an object, a situation often arises when such image does not have the completeness of information required to achieve its goals and limits the analysis to a schematic image or a truncated viewing angle. Unlike a two-dimensional flat image, three-dimensional stereo allows exploring more effectively and modeling objects with a developed spatial structure or interpreting pictures, made in the course of the experiment from different angles at specific points in time and combined into an animation.

The representation of numerical simulation results that display the flow of complex physical processes over time or show the operation of complex technical installations in threedimensional representation allows implementing a wide range of features. Firstly, a threedimensional representation of the process development over time helps to verify the compu- 
tational model and applied algorithms. Secondly, it provides the observer with a full understanding of the simulated phenomenon. Finally, it popularizes the research and its results for society, including for decision makers. All of the above makes theoretical and practical developments in the field of building stereo representations very important.

With the advent and development of stereo installations, more and more works devoted to the construction of stereo images began to appear. Specific problems that arise when using a computer system to generate and visualize a composite multi-screen stereo frame, and methods for solving such problems are described in sufficient detail in [1-3]. At first, most research in the field of stereo images related to the tasks of building virtual reality, simulators and trainers, as well as training systems. However, later the issue of building presentation complexes became very relevant, which also allowed demonstrating the results of scientific research in stereoscopic mode. These works include works [3-10]. In [3], the results of displaying a Supernova explosion in stereo mode are presented. The work [7] is devoted to the creation of a computational technology for modeling the operation of a three-dimensional node of power plant blades when the flow of a viscous compressible heat-conducting gas flows around. Currently, the problems of constructing stereo images are considered in a wide variety of research areas [3-9].

However, when constructing a stereo image of the scientific calculations' results, a rather important particular structural subproblem arises. As a rule, the standard presentation of a scientific report includes not only visualization of complex geometry or fields of physical quantities, but also explanations, usually represented by text and formulas. If for geometries and physical quantities the issues of constructing stereo representations are sufficiently developed, then the tasks of combining them with stereo images of texts and formulas with sufficient expressiveness and the necessary stereo effect are still open.

As for the development of specific practical approaches in the field of combining images and textual information to it, quite a few works have been devoted to this topic. Only the work [10] can be mentioned here describing a study conducted in Japan on the perception of stereo images of fonts on the screens of stereoscopic mobile devices. However, this research was more social in nature rather than providing recipes for constructing stereo images of texts and formulas.

Construction of stereo images is possible using a fairly wide range of different technologies: from classical stereoscopy to the use of depth maps. The choice of technology depends on the type of affordable stereo system, the field of application, and directly on the goals of creating the stereo image.

Unlike the film industry in scientific research topics, the given area is still developing and adapting to its needs and specific requirements. Precisely depending on the transmission accuracy of the original images, values of the required stereo effect, correspondence to the research topic, one or another technology for creating stereo images is defined and selected. This work is a continuation of the cycle of research works [1-3,7,11-14] carried out at the Keldysh Institute of Applied Mathematics on the basis of existing stereo devices. The main type of device, that the stereo imaging software system described in this paper is designed to work with, is the Dimenco DM654MAS autostereoscopic monitor. Autostereoscopic monitors provide the display of stereo images without having to track the observer's position. Usually these monitors allow observing stereo images, providing a few fixed segments in the observation space. The spectator can move between segments, getting the opportunity to view the displayed object in 3D from different angles of view. The operation principle of autostereoscopic monitor is the use of parallax partitions or Fresnel lenses installed behind the protective glass of the screen, which gives it one of the most important advantages: the image display does not require the viewer to have special glasses or other devices.

An autostereoscopic monitor is capable of demonstrating the visualization object in two ways: either using a composite frame containing views of the visualization object from different angles that form a certain sector of the review- this method is called multi-view - or using depth maps. 
In $[12,13]$, the step-by-step process of developing such a technology for creating stereo images matched with stereo text was considered in detail . These stereo images were constructed on the base of multi-view technology. This technology allows achieving the highest stereo effect to visualize the results of mathematical modeling which is engaged at the Keldysh Institute of Applied Mathematics[7,14,15].

However, it is worth noting that until now, the construction of stereo frames was carried out almost manually, even if using a well-established technology, by applying various programs and sequential processing of each frame with setting and configuring all the necessary parameters. This process required a large amount of time and a high degree of accuracy and care.

This work is devoted to the development of the task of automating this process and providing automatic frame generation where multiple objects or inscriptions could be placed, each of them has its own stereo base.

\section{Methods for combination graphic and text information in a multi-view for an autostereoscopic monitor}

Autostereoscopic monitor has the ability to demonstrate the visualized object using a composite frame containing views of the visualization object at different angles, which together form a certain sector of the review. Usually, there are nine types. These nine species form eight stereo pairs $([1 / 2],[2 / 3],[3 / 4], \ldots,[8 / 9])$, however, the viewer can only watch one of the stereo pairs in one position, depending being in a certain corner sector of observation. When the observer moves from sector to sector, he gets information about the object using all nine angles, that is, as if inspecting at the visualized object from different sides.

When building a multi-view stereo image, nine frames are connected into one image based on the $3 \times 3$ matrix principle. In the first and last (ninth) frames, the represented object is in its extreme positions. In the first frame, the object is in its rightmost position, in the last one - in the far left. Next in the middle frames, respectively from the second to the eighth, with certain equal intervals, it shifts to the left, until it reaches the leftmost position. Herewith, in each frame, the object turns progressively at the same angle and it is shifted by the specified interval. This allows achieving the most effective result when building a stereo image of an object. With too large parameters of the angular shift, artifacts occur: the observer has a feeling that the image in front of him is blurring at the transitions from one stereo pair to another, and at the slightest change of position, the observer notices the highlights and borders of each of the stereopairs. Similar phenomena can cause a certain discomfort in the observer, which interferes with work with stereo images and adequate perception of visual information on a stereo frame. The problems of visual perception discomfort in virtual and mixed reality systems are described in detail in [17].

After solving the problem of creating a multi-view representation of an object, in the investigated case of stereo labels, in the quality separate frames in a stereo presentation or stereo film, an equally important sub-task was considered- matching the image and text information in one frame. Currently, in the case of demonstrating scientific results to observers, very many objects require accompanying information situated directly on the same frame as the image. In many cases, the caption and the object can not be distributed into different frames, because they constitute a single logical display of information.

To construct such a stereo image in which both the object and the caption to it would be combined, an image matrix is used (Fig. 1), to which three-dimensional letters were added and sequentially shifted and rotated at each frame by the same interval (angular stereobase of the object), previously calculated experimentally. The result was a stereo image with a threedimensional caption, where the stereo effect was pronounced.

Figure 1 presents an image of the results of modeling supersonic flow around a cone at an angle of attack with the corresponding caption . This is one of the results of previous research- a multi-view image of the results of modeling the supersonic flow around the cone at an angle 
of attack with the corresponding inscription [16]. Here, the very image of the modeled cone and separate inscriptions to it are matched. Each of them is rotated to its own experimentally determined angle. As shown in the figure, then a matrix of images is compiled, which in turn make up a single stereo image. Ultimately, the inscription was placed over the cone, but behind its point, which in turn, was perceived by the audience as protruding from the screen by several tens of centimeters.

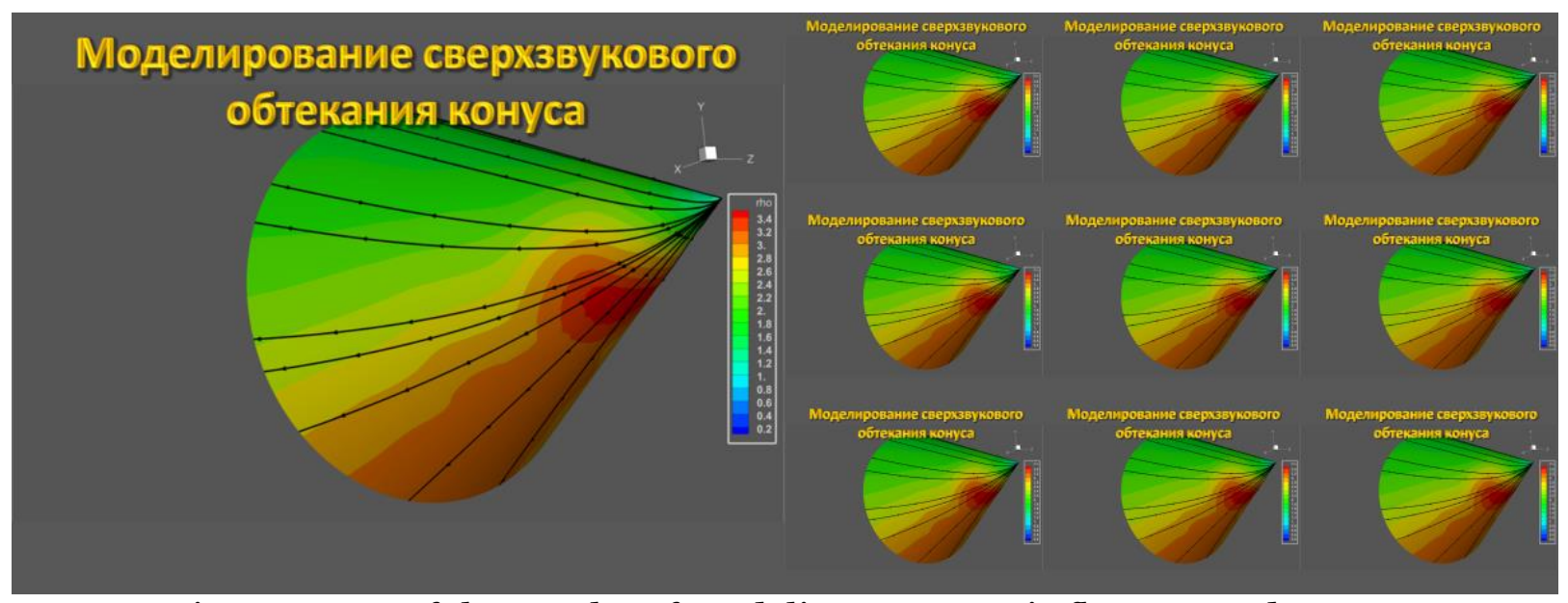

Fig. 1. Image of the results of modeling supersonic flow around a cone with the corresponding caption [16].

In the process of research, the following technology for constructing a similar stereo frame was worked out, which is performed manually by the user:

1) The rotation of the object (the result of visualization of scientific computations) on the $Z$ axis in the Tecplot program by a given degree and subsequent saving of the frame with each rotation;

2) Creating 9 frames for every rotation : 1st frame - extreme left position , 9 - the extreme right;

3) Adding the required text (having volume and clarity) for each frame;

4) Consecutive rotation of the text by the required fraction of a degree in each frame;

5) Stitching all frames into an image matrix in certain sequence.

Building manually nine versions of such a stereo frame, which there are present several objects in, each of which has its own stereo base, takes a lot of time. It is necessary to build nine views for each object, and then match them, without violating the integrity of the final stereo image. The main difficulty in building a stereo image with matching several stereo objects is the need for multiple repetitions of the same operation while generating the same stereo image.Multi-view StereoMaker software package was developed to automate this process.

\section{Multi-view StereoMaker software package}

Multi-view StereoMaker program represents a software package, allowing to create a set of nine images / representations of rotation of three-dimensional objects on a given angular stereo base, and then get their union, matched into one image.

Multi-view StereoMaker was implemented in Python 3.6. In view of the fact that there were no restrictions on the speed of the software package, when choosing given language, the following criteria as cross-platform, simplicity of the language and its broad support for optional ready functionality were taken into account. The program is designed to work with arbitrary $3 \mathrm{D}$ objects with specified parameters. As such $3 \mathrm{D}$ objects can be the results of scientific calculations and accompanying information to them, such as accompanying captions, formulas, additional images. Because of the visualization of the scientific calculations used was carried out in the Tecplot software package, then Multi-view StereoMaker additionally organizes the implementation of interaction with this software package. The program Blender was chosen 
as a software package for generating arbitrary $3 \mathrm{D}$ images, which is open source and free software with extensive support for the python language. A screenshot of the Multi-view StereoMaker interface is shown in figure 2.

Among the main functions of the program, the particularly important are selection of the stereo image generation algorithm for each object, the ability to preview the generated images according to the specified parameters for each object, setting the parameters of generated images on top of each other by the user- specified coordinates and also the selection of the way to save the final result.

Among the algorithms for generating stereo images, there is a category of "custom images", where the user can respectively specify the directory where his images are. When choosing the Tecplot category, the user must specify file in plt format with necessary model and when setting the necessary parameters specify the size and width of the output image, the initial rotation angle, the number of rotations, the object rotation angle, the axis of rotation.

The last category - the Blender algorithm - assumes generating a $3 \mathrm{D}$ object either from the original user image in the .svg format, or by using internal text generation, as well as indication of additional parameters such as the color and depth of the $3 \mathrm{D}$ object, its scale, size and width of the output image, starting angle of rotation, number of turns, axis of rotation, etc.

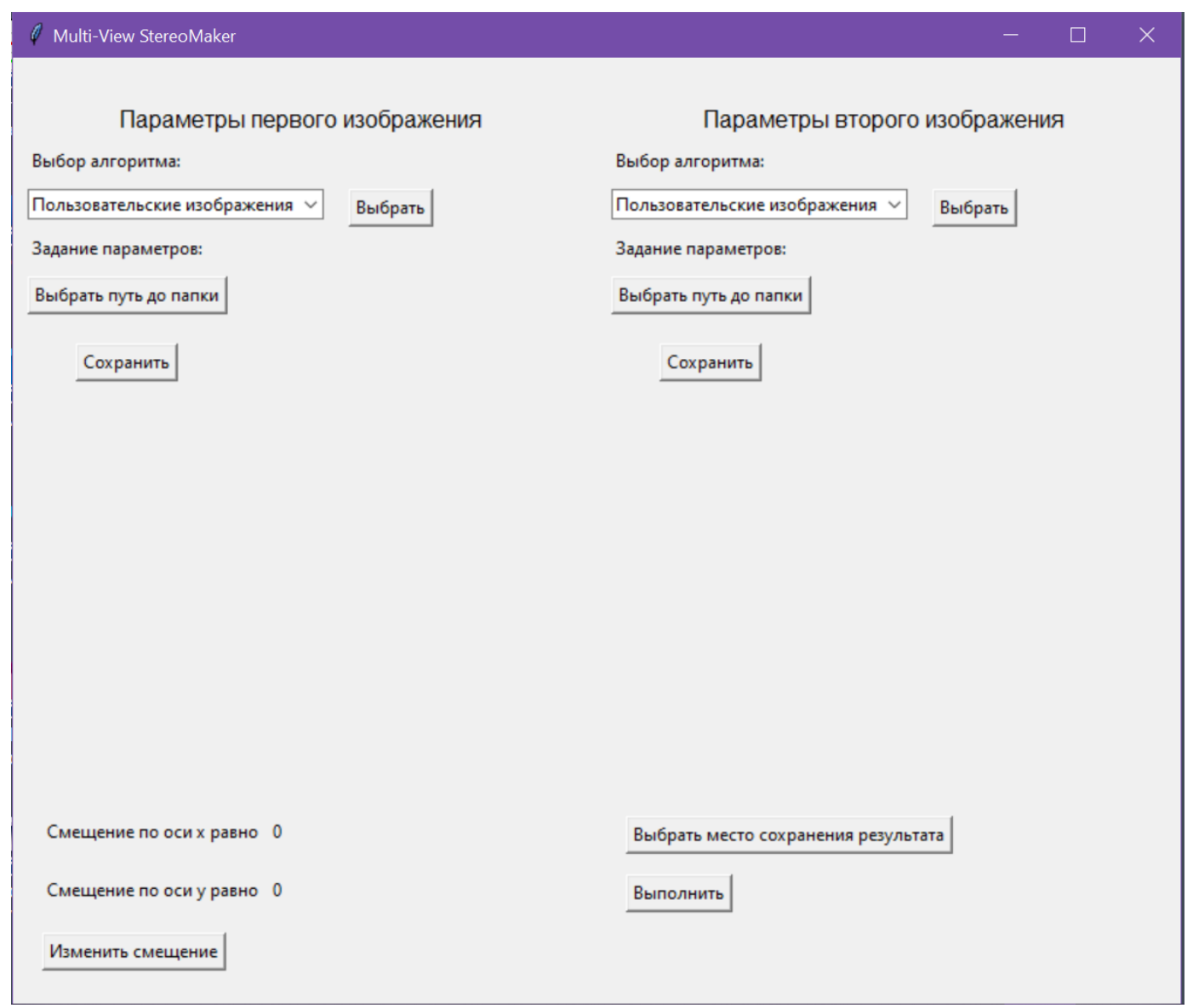

Fig. 2. Screenshot of the interface of the Multi-view StereoMaker software package.

The program includes the following components, shown in the diagram (Fig. 3.). The user must enter the parameters using the provided graphical interface (GUI).The graphical interface allows referring to the generated images preview subsystem for each object. The parameters entered by the user are transmitted to the preliminary data subsystem, which creates a directory system for saving the results of algorithms. Then the parameters are passed to the algorithm generator. 


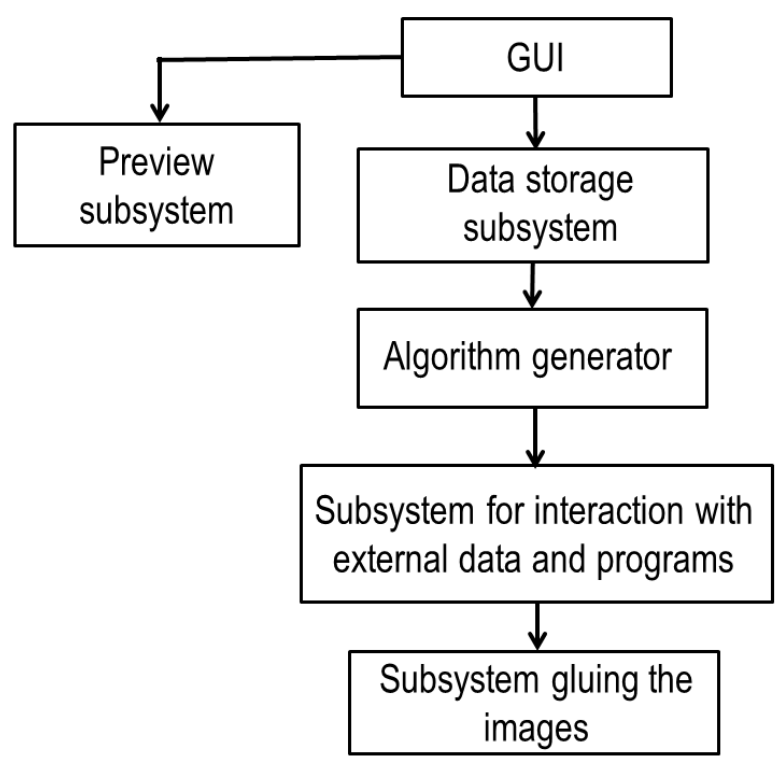

Fig. 3. Organization diagram of the Multi-view StereoMaker software package.

The algorithm generator generates the appropriate algorithms for the external programs' operation (Tecplot, Blender), after which the obtained algorithms and parameters are transferred to the subsystem of interaction with external programs and data. In the case of transferring custom images, given subsystem will copy the custom images to the resulting directory and transfer them to the image gluing subsystem. In all other cases, the given subsystem will launch external programs with the algorithms specified for them. Then there is a call to the subsystem of gluing images, which performs multiple superposition of a number of images formed for two objects. The result of the program's work is a directory containing algorithms formed for a specific case for external programs, directories with generated images for objects with user-specified parameters, and a directory with the final result.

Figure 4 presents the results of the operation of the Multi-view StereoMaker software package, demonstrating three-dimensional visualization of multicriteria alternatives using the histograms described in [18].

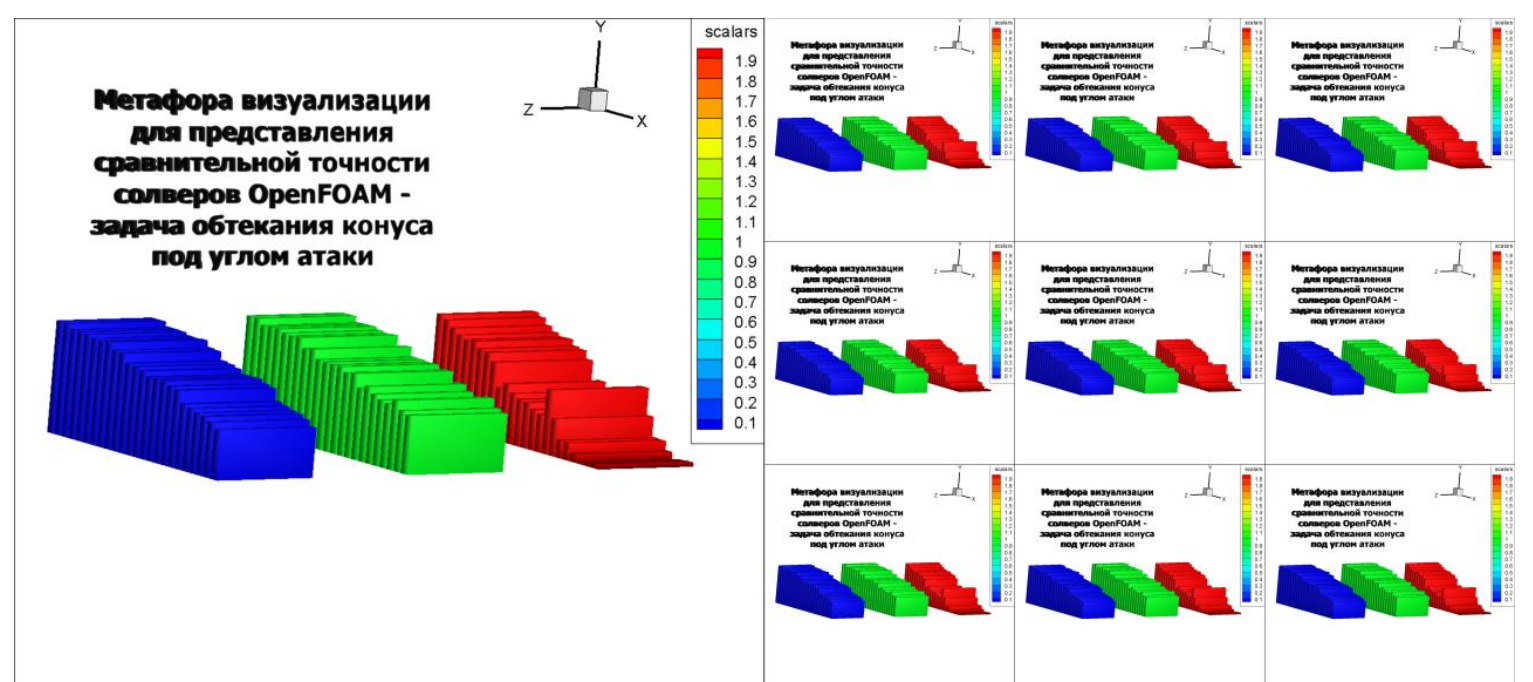

Fig. 4. $3 \mathrm{D}$ visualization of three multi-criteria alternatives using histograms.

This project is not completed. Only the first version of the developed program is presented. It is planned to further develop and improve the program for combining multiple objects and interactively placing them in a stereo frame with the simultaneous generation of nine views for each. The additional options for recording statistical data will also be developed. 


\section{Conclusion}

The results of constructing a stereo image for an autostereoscopic monitor by the method of multi-view representation using the Multi-view StereoMaker software package are presented. In the course of practical experiments, an optimal technology for constructing multi-view stereo images of the results of solving mathematical modeling problems has been developed.

The goal of this work is to automate manual processes and development of a program for constructing stereo images with textual accompaniment for creating stereo presentations. In the long term, further debugging and testing of the program and automatic conversion to the required autostereoscopic monitor format, as well as solving the problem of interactively placing objects in one stereo frame with the simultaneous generation of nine views for each are expected.

\section{References}

[1] Synthesis of photorealistic three-dimensional images in modern presentation systems / Andreev S.V.[et al] // Software\&Systems 2007. № 3. p. 37-40. [in Russian]. Hardwaresoftware complex of $3 \mathrm{D}$ presentations based on a virtual studio and virtual environment / Vandanov V.G. [et al] // Proceedings of the 1-st international conference "3D visualization of scientific, technical and social reality. Cluster technologies of modeling» Izhevsk. 2009. P.7377. [in Russian].

[2] Generation of Stereo-Presentations in Photorealistic Rendering and Scientific Visualization /Andreev S.V.[et al] // Keldysh Institute preprints, 2010. № 61.16 p. http://library.keldysh.ru/preprint.asp?id=2010-61[in Russian].

[3] Andreev S., Filina A. Using stereo presentations for visualization of scientific calculations results / Scientific Visualization. 2012. v.4. № 1. p.12-21

[4] Hardware-software complex of $3 \mathrm{D}$ presentations based on a virtual studio and virtual environment / Vandanov V.G. [et al] // Proceedings of the 1-st international conference "3D visualization of scientific, technical and social reality. Cluster technologies of model-ing» Izhevsk. 2009. P.73-77. [in Russian].

[5] Mezhenin A.V., Tozik V.G. 3D Visualization using the stereo image effect // Proceedings of the 2-nd international conference $43 \mathrm{D}$ visualization of scientific, technical and social reality. Cluster technologies of modeling» Izhevsk. 2010. [in Russian].

[6] Mikhaylyuk M.V., Huraskin I.A. Synthesis of stereo images for virtual reality systems using an optical tracking system / Software\&Systems 2006. № 3. p. 10-14. [in Russian].

[7] Andreev S.V. [et al] Modelling and visualisation of blade assembly with complicated shape for power turbine / Scientific Visualization. 2015. v.7. № 4. p.1-12

[8] Torgashev M.A., P.Y. Timokhin. The technology of stereo video files' synthesis for the system of $3 \mathrm{D}$ real-time visualization. Software Products and Systems, 2012, № 3, pp. 74-80. [In Russian]

[9] Mikhaylyuk M.V., Maltsev A.V. Timokhin P.Yu. The methods of 3D stereo visualizationof data obtainedin simulation of unstable oil displacement from porous media / Proceedings of Scientific Research Institute for System Analysis RAS, 2018, v.8, N 2, p. 125-129. [in Russian].

[10] Visibility Experiment and Evaluation of 3D Character Representation on Mobile Displays / Hiromu Ishio [et al.] // C. Stephanidis (Ed.): Posters, Part II, HCII 2011, CCIS 174, pp. 46-51, 2011.

[11] Andreev S.V., Bondareva N.A. Constructing a representation of textual information in stereo presentations // Proceedings of the 28-th International Conference of Computer Graphics and Vision GraphiCon-2018, Tomsk, Russia, 23-28 September 2018, p. 86-89. [in Russian].

[12] S.V.Andreev, A.E.Bondarev, V.A.Galaktionov, N.A.Bondareva (2018) The problems of stereo animations construction on modern stereo devices. Scientific Visualization 10.4: 40 52, DOI: $10.26583 /$ sv.10.4.04 
[13] S.V. Andreev, N.A. Bondareva, E.Yu. Denisov. Stereo Presentations Problems of Textual information on an Autostereoscopic Monitor (2019). Scientific Visualization 11.4: 90 - 101, DOI: $10.26583 / \mathrm{sv} .11 .4 .08$

[14] S.V. Andreev, A.E. Bondarev, N.A. Bondareva. Stereo images of error surfaces in problems of numerical methods verification (2020). Научная визуализация 12.2: 151 - 157, DOI: 10.26583/sv.12.2.12

[15] Stereo animation "Modeling and visualization of the work of a power plant having complex shape in the stereo animation mode". https://yadi.sk/i/3CcYhlMB3TCH4r (Accessed 01.11.2020).

[16] S.V. Andreev, A.E. Bondarev, N.A. Bondareva. Stereoscopic construction of textual information in presentations of research results on an autostereoscopic monitor (2020). Scientific Visualization 12.1: 132 - 139, DOI: 10.26583/sv.12.1.12

[17] A.D. Zhdanov, D.D. Zhdanov, N.N. Bogdanov, I.S. Potemin,V.A. Galaktionov, and M.I. Sorokin. Discomfort of Visual Perception in Virtual and Mixed Reality Systems // Programming and Computer Software, 2019, Vol. 45, No. 4, pp. 147-155. DOI: 10.1134/So36176881904011X

[18] A.A. Zakharova, D.A. Korostelyov. Application of Visual Analytics Methods to Reduce the Dimensionality of Decision-making Problems (2020). Scientific Visualization 12.4: 23 32, DOI: $10.26583 /$ sv.12.4.03 